Grzegorz Chromik

ORCID: 0000-0001-9318-6761

Jagiellonen-Universität, Kraków

DOI: $10.19195 / 0435-5865.143 .9$

\title{
Die Entwicklung der Familiennamen der Seibersdorfer Auswanderer in Anhalt/Hołdunów
}

\begin{abstract}
s
Im Jahr 1770 wanderten über 300 deutsche Einwohner des Dorfes Seibersdorf/Kozy in Kleinpolen nach Preußisch-Schlesien aus und gründeten somit eine neue Sprachinsel (Anhalt/Hołdunów). Der vorliegende Beitrag behandelt das Thema der Seibersdorfer Familiennamen, die vor der Auswanderung durch des Deutschen unkundige polnische Schreiber einerseits entstellt, andererseits aber in ihrer dialektalen Form konserviert wurden (etwa Bayger statt Berger, Wayner statt Wagner). Nach der Auswanderung wurden die Namen durch die preußischen Beamten richtiggestellt, oder in manchen Fällen ,verschlimmbessert" (Bayger wurde wieder Berger, aber aus Englert wurde Engel statt richtig Engelhard).
\end{abstract}

Schlüsselwörter: Deutsche Sprachinsel Bielitz, Deutsche Sprachinsel Anhalt, schlesischer Dialekt, Familiennamen

\section{The development of the surnames of the Seibersdorf emigrants to Anhalt/Hołdunów}

In 1770 three hundred German inhabitants of the village of Seibersdorf/Kozy in Lesser Poland emigrated to Prussian Silesia and founded there a new language island (Anhalt/Hołdunów). This article deals with the surnames of the emigrants. Before the emigration the Polish writers who did not know German disfigured them or conserved the dialectal form (for example Bayger instead of Berger, Wayner instead of Wagner). After the emigration the Prussian authorities corrected the surnames or sometimes made them worse by trying to correct it (Bayger became Berger but Englert became Engel instead of Engelhard).

Keywords: German Language Island Bielitz, German Language Island Anhalt, Silesian German dialect, surnames

Grzegorz Chromik, Uniwersytet Jagielloński, Instytut Filologii Germańskiej, Al. Mickiewicza 9a, pok. 312, 31-120 Kraków, Polen, E-Mail: Gmchromik@yahoo.com

Received: 1.10.2017, accepted: 18.05.2018

Germanica Wratislaviensia 143, 2018

(C) for this edition by CNS 
Es passiert recht selten, dass eine geschlossene Gruppe von einigen Hundert Personen auswandert und sowohl in ihrer alten als auch neuen Heimat historische Quellen vorhanden sind, die sprachhistorische Untersuchungen erlauben. So ein Fall war die Umsiedlungsaktion der deutschen Einwohner des Dorfes Seibersdorf/ Kozy in Kleinpolen, die 1770 auf Einladung Friedrich des Großen nach PreuBisch-Schlesien zogen und somit eine neue Sprachinsel gründeten (Anhalt/Holdunów). Der vorliegende Beitrag behandelt das Thema der Seibersdorfer Familiennamen, die vor der Auswanderung durch des Deutschen unkundige Schreiber einerseits entstellt, andererseits aber in ihrer dialektalen Form konserviert wurden. Nach der Auswanderung wurden die Namen durch die preußischen Beamten richtiggestellt, oder in manchen Fällen ,verschlimmbessert“. Berücksichtigt wurden jedoch auch diejenigen Seibersdorfer Familiennamen, deren Träger nicht an der Auswanderung teilnahmen, die aber in den Seibersdorfer Quellen zu finden sind und als Beweise dialektaler Verhältnisse dienen können.

\section{Seibersdorf/Kozy als Teil der ehemaligen Deutschen Sprachinsel Bielitz}

Die Deutsche Sprachinsel Bielitz ist ein gut bekanntes und erforschtes Kulturphänomen. Entstanden im 13. Jh., überdauerte sie bis zur Vertreibung 1945. Wegen der politischen Verhältnisse ist ihre Entwicklung aber unterschiedlich verlaufen. Gegründet wurde sie während der Unabhängigkeit der schlesischen Herzogtümer, im Herzogtum Oppeln-Ratibor, von dem sich rasch das Herzogtum Teschen absonderte, das auch das Auschwitzer Gebiet umfasste. Um das Jahr 1316 wurde der Auschwitzer Teil unabhängig. Seibersdorf lag eben in diesem Teil, der 1456 an den polnischen König verkauft wurde. Es lässt sich nicht mit Sicherheit sagen, wann die Reformation in Seibersdorf und der Gegend Fuß fasste. Erste Erwähnungen der Reformation in der Woiwodschaft Krakau stammen von 1529. Die Konversion zum Kalvinismus muss also Mitte des 16. Jh. erfolgt sein. Für die späteren Ereignisse, d.h. die Auswanderung der Seibersdorfer, waren religiöse Gründe ausschlaggebend. Im Jahr 1658 wurde die von den Seibersdorfer Protestanten, die meistens reformierten Konfessionen angehörten, erbaute hölzerne Kirche vom Krakauer Bischof beschlagnahmt, neu geweiht und mit einem katholischen Pfarrer besetzt. Ein katholischer Grundherr erlaubte ihnen jedoch um 1680, einen Pastor zu berufen und in einem Stadel Gottesdienste abzuhalten. Die Erlaubnis wurden ihnen später entzogen, so dass sie sich im Wald an der Grenze zu Alzen/Hałcnów zu Gottesdiensten versammelten. Die Trauungen und Taufen wurden vom katholischen Pfarrer durchgeführt. Trotz der Gegenreformation gaben nur wenige Deutsche aus dem Ort den reformierten Glauben auf (im Dorf wohnte auch eine polnische Minderheit, zumeist Katholiken). Auf die Ausübung protestantischer religiöser Praktiken in Häusern 
standen Prügelstrafen. Der Wechsel der Grundherren zur katholischen Familie Jordan verursachte, dass sich zur religiösen Verfolgungen noch die wirtschaftlichen gesellten. Oft wurden die Landwirte ihrer Höfe verwiesen. Die Arbeit auf dem Gutshof dauerte die ganze Woche, darüber hinaus mussten die Bauern im Gebirge als Köhler dienen. Eine Hoffnung für die Seibersdorfer brachte erst die Eroberung eines großen Teils von Schlesien durch das protestantische Preußen im Jahr 1740, wodurch in diesem Gebiet die Gegenreformation ganz aufhörte. ${ }^{1}$

\section{Die Auswanderung der Seibersdorfer nach Preußisch-Schlesien und die Entstehung der deutschen Sprachinsel Anhalt}

Um das Jahr 1754 begannen die Seibersdorfer Protestanten, reformierte Gottesdienste im schon damals preußischen Pless/Pszczyna zu besuchen, wie sie es mit den dortigen Geistlichen ausgemacht hatten. Im Herbst 1765 wanderten 48 Personen aus Seibersdorf in die Standesherrschaft Pless aus, dessen Eigentümer Fürst Friedrich Erdmann von Anhalt-Cöthen war. Sie ließen sich in Lendzin/Lędziny nieder. Die beginnende Auswanderung der Seibersdorfer traf auf die Kolonisationsbestrebungen des preußischen Königs Friedrichs des Großen. Am 5.01.1770 erging ein königliches Edikt, in dem fremden Handwerkern, die sich in Preußen niederlassen würden, Begünstigungen, Freiheiten und Ausnahmerechte zugesichert wurden. Eine besondere Begünstigung genossen die Leinweber, d.h. eine zehnjährige Befreiung von Steuern, Militärdienst und Zöllen. Die Leinweberei war ein beliebtes Handwerk in Seibersdorf. Der Seibersdorfer Vogt Johann Mandzla und der Landrat von Pless, Freiherr von Skrbensky, begannen Umsiedlungsverhandlungen, die endlich im April nach manchen Verpflichtungen des Plesser Grundherrn und des Königs abgeschlossen wurden. Man befürchtete, dass der Seibersdorfer Grundherr Jordan mit allen Mitteln versuchen würde, die Auswanderung zu vereiteln. Der Preußenkönig erließ höchstpersönlich den Befehl, die Seibersdorfer Einwanderer militärisch zu beschützen. Zum Ausführer dieser Aufgabe wurde Leutnant Georg von Woyrsch, der am 25.05.1770 mit einer 70 Mann starken Schwadron Husaren die polnische Grenze überschritt und Seibersdorf besetzte. Eine zweite Schwadron blieb als Rückendeckung auf preußischen Boden in Miedźna. Die Preußen brachten 220 Wagen mit, zu denen sich die Auswanderer

${ }^{1}$ Wackwitz (1932: 16-23). Alle Nachrichten zur Geschichte der Seibersdorfer Auswanderung sind in den Akten des Landratsamtes Pless und des Fürstentums Pless zu finden - Archiwum Państwowe w Katowicach, Oddział w Pszczynie (Staatsarchiv Kattowitz, Filiale Pless), Bestand Archiwum Książąt Pszczyńskich [Archiv der Fürsten von Pless], Signatur XIII 606, 607; Bestand Landratura [Landratsamt], Signatur 122. Sie wurden von A. Wackwitz und später auch von Adam Malina benützt. 
mit ihren eigenen Fahrzeugen gesellten. Der Abgang geschah ohne ernstzunehmenden Widerstand der Polen und völlig nach Plan. Am Abend desselben Tages überschritt die Kolonne die polnisch-preußische Grenze bei Miedźna. ${ }^{2}$ Sie zählte 303 Personen und einige Hundert Stück Vieh. Unter den Auswanderern befand sich ein Katholik, Komendera, der eine protestantische Frau geheiratet hatte. Der Landrat berichtete nach dem erfolgreichen Unternehmen, dass alle Einwanderer des Deutschen mächtig seien, da sie für die preußischen Behörden ja polnische Immigranten waren ${ }^{3}$. Die Einwanderer trugen folgende Namen: Banert, Berger, Borusiak, Czauderna, Englert, Hoinkes, Herma, Klotzky, Komendera, Mansla/ Mansel, Schön, Steffke/Stewki, Wagner, Zenger.

\section{Seibersdorf und die Seibersdorfer nach dem Exodus der Protestanten}

Der Grundherr schätze seinen durch die Auswanderung verursachten Schaden auf 50000 Zloty ein. Ein Teil der dagebliebenen Einwohner war mit den Auswanderern verwandt, wovon die heute noch gebräuchlichen deutschen Namen zeugen (Honkisz, Hankus, Nycz, Stefko, Komędera, Herma, Czaderna). ${ }^{4}$

Für die Ex-Seiberdorfer wurde 1770 ein neues Dorf auf dem Gebiet eines parzellierten Vorwerks in Lendzin/Lędziny angelegt, das als eine Tochtersiedlung der Bielitzer Sprachinsel gelten kann. Nach dem Geschlecht der Plesser Grundherren erhielt es den Namen Anhalt. Da es nicht für alle Siedler ausreichte, wurde in einer Entfernung von 500 Metern 1775 Neu-Anhalt angelegt. ${ }^{5}$ Ursprünglich sollte die Kolonie Haltan heißen. Die polnische Form Hołdunów ist eine Entstellung dieses ursprünglichen Namens. ${ }^{6}$ Anfang des 19. Jhs. entstand noch die Kolonie Gatsch/ Gać, deren Einwohner alle Seibersdorfer Abstammung waren. ${ }^{7}$

\section{Deutsch-polnischer Sprachkontakt in Seibersdorf}

Inwieweit die Seibersdorfer die polnische Sprache beherrschten und ihre katholischen Pfarrer die deutsche, ist nicht bekannt. Im Bericht des Plesser Landrats nach der erfolgten Einwanderung nach Preußen war lediglich davon die Rede, dass sie alle Deutsch verstanden. Der Anhalter Religionslehrer Richter beklagte sich 1802,

2 Wackwitz (1932: 24-33).

${ }^{3}$ Wackwitz (1932: 34).

${ }^{4}$ http://gimnazjum.nexcom.pl/index.php/olimpiady-i-konkursy, http://www.osp.kozy.pl (28.09.2017), Wackwitz (1932: 35).

5 Wackwitz (1932: 37, 45-46).

6 Wackwitz (1932: 50).

7 Wackwitz (1932: 59). 
dass die Kinder nur ihren Dialekt sprechen und die Hochsprache nicht verstehen würden, ebenso wenig wie der Lehrer ihre Sprache. Auch des Polnischen seien sie nicht kundig gewesen. ${ }^{8}$ Eindeutig deutsch war ihre Kultur bis zur Vertreibung aus der Sprachinsel Anhalt im Jahr 1945. Interessant wäre zu erfahren, ob sie die polonisierten Formen ihrer Namen als richtig empfanden (etwa Mandzla, Banek, Gietler, Ankusz, Klocek, Lenier, Stefko). Aus dem oben Gesagten resultiert, dass die Seibersdorfer wenig Kontakt zum Polnischen hatten und die polonisierten Namenformen eher als etwas Amtliches betrachteten.

\section{Zur Methode der Namenforschung, Quellenlage}

Die ältesten Quellen zu den Familiennamen der Seibersdorfer stammen aus dem 17. Jh. In der Literatur wird auch ein Gemeindebuch aus Kunzendorf/Lipnik aus dem 16. Jh. erwähnt, das heute verschollen ist. Die Kirchenmatrikeln reichen in das Jahr 1640 zurück. Die Quellen aus Anhalt sind eine nahtlose Fortsetzung zu den Informationen über die gleichen Personen. Im Plesser Staatsarchiv werden zwei umfangreiche Konvolute Akten zu der Siedlungsaktion des Jahres 1770 aufbewahrt, eine weitere Quelle bieten die Kirchenmatrikeln der reformierten Pfarrei Anhalt, die heute im Internet einsehbar sind. Eine Hilfe ist der Vergleich dieser Familiennamen mit dem Schlesischen Namensbuch von Hans Bahlow (1953) und anderen Bearbeitungen zur schlesischen und inseldeutschen Namenkunde. Bis dato wurde der Dialekt der Anhalter Siedler nicht erforscht. Der Vergleich der Einträge in die katholischen Kirchenmatrikeln von Seibersdorf und der Siedlerlisten in Anhalt sowie den schon oben erwähnten evangelischen Kirchenmatrikeln von Anhalt erlaubt, die Entwicklung der Familiennamen zu verfolgen, weil man in allen drei Quellen mit denselben Personen zu tun hat.

\section{Die Entwicklung der häufigsten Seibersdorfer und Anhalter Familiennamen}

In diesem Abschnitt wird die historische Entwicklung der in Seibersdorf und Anhalt gebräuchlichen Familiennamen dargestellt, ausgehend von den ältesten Erwähnungen bis hin zu den Formen, die kurz vor der amtlichen „Versteinerung“ der Familiennamen wegen der Gründung der Standesamtswesens in Preußen 1875 im Gebrauch waren. Es wird die Etymologie dieser Namen angegeben, die in den Quellen belegten Formen und der Versuch, die Veränderungen zu erklären. Alle nicht explizit genannten Quellen für die Belege vor 1770 sind die katholischen Kirchenmatrikeln von Seibersdorf.

${ }^{8}$ Wackwitz (1932: 164). 


\section{Banert}

Kurzform zu Bernhard. ${ }^{9}$ In Seibersdorf schon 1584 belegt. ${ }^{10}$

17. Jh.: Banert 1692, Barnet 1696

Vor der Auswanderung: Banet 1757, Banek 1770

Nach der Auswanderung nach Anhalt: Banert

Nach 1870: Banert

Durch die polnischen Geistlichen offensichtlich entstellt, wurde der Name nach der Auswanderung nach Preußen ,rekonstruiert“.

\section{Berger}

„[V]on der Wohstätte auf oder an einem Berge [...]"11

17. Jh.: Baygerin 1659, Bager 1660, Bagier 1666, 1668, 1670, 1672, 1682; Bargier 1666, Balger 1696, Barger 1696, Baiger 1697

Vor der Auswanderung: Bagier 1769, Beyger 1770

Unmittelbar nach der Auswanderung nach Anhalt, 1770: Beiger korrigiert auf Berger

Nach 1875: Berger

Nach Bukowski (1860: 169) war 'Barg' die Bielitzer Aussprache des Wortes Berg. Deswegen ist die Aussprache dieses Familiennamens als Barger durchaus möglich. Die polnischen Pfarrer schrieben diesen Namen einfach phonetisch, während ihn die preußischen Beamten ,richtigstellten“. Noch in der 1930er Jahren wurde der Name im Dialekt „Bayger“ ausgesprochen.

Bittner

Eigentlich Büttner 'Fassbinder'.

17. Jh.: Bytnerowa 1658.

An der Auswanderung nach Anhalt nahmen keine Träger dieses Namens teil.

Bräuer

Berufsbezeichung.

17. Jh.: Broierowa 1672, Broier 1675

An der Auswanderung nach Anhalt nahmen keine Träger dieses Namens teil.

\section{Czauderna}

Nicht klar deutbar. Wahrscheinlich kein deutscher Name. Auch im Polnischen bedeutet dieses Wort nichts.

17. Jh.: Czauderna 1658 u.a.

Vor der Auswanderung: Czałderna 1770

Unmittelbar nach der Auswanderung nach Anhalt, 1770: Czauderna

\footnotetext{
${ }^{9}$ Kuhn (1981: 91).

${ }^{10}$ Kuhn (1981: 51).

11 Bahlow (1953: 79).
} 
Nach 1870: Czauderna.

Interessanterweise keine orthographische Verdeutschung des Namens z.B. durch die Verwendung des Graphems $t s c h$.

Englert

Der Name ist in Seibersdorf schon im 16. Jh. belegt. Eine Kurzform des altdeutschen Namens Engelhard.

17. Jh.: Englit 1659, Englet 1659, Englert 1660, 1681, Enlert, Engelert 1661, Enlet 1662

Vor der Auswanderung: Engel 1769

Nach der Auswanderung nach Anhalt: Englert

Nach 1870: Engel

Die preußischen Beamten machten durch Volksetymologie aus dem altdeutschen Engelhard einen Engel der Christen.

Feikis

Nach Bahlow Verdeutschung eines slawischen Namens mit dem Stamm woj „Mann“, „Krieger“.

17. Jh.: Feykiß 1658

An der Auswanderung nach Anhalt nahmen keine Träger dieses Namens teil.

Fuchs

Klar deutbarer Übername: der Fuchs.

17. Jh.: Fox 1697, Fux 1659

An der Auswanderung nach Anhalt nahmen keine Träger dieses Namens teil.

Getfert

Eine entstellte Form des altdeutschen Namens Gottfried. Interessanterweise war dies einem Schreiber im Jahr 1661 klar.

17. Jh.: Getwelt 1660, Getfedowa 1662, Getfetowna 1686, Goftridowa 1661

Großman

Klar deutbarer Übername.

17. Jh. - Großman Johann und Susanna geb. Englert 1669, 1672, Großman Johann 1676

An der Auswanderung nach Anhalt nahmen keine Träger dieses Namens teil.

Gürtler

Eine Berufsbezeichnung.

17. Jh.: Gietler 1694

An der Auswanderung nach Anhalt nahmen keine Träger dieses Namens teil. Hoinkes 
Eine Variation des Vornamens Hans (Johannes). Die Endungen -usch und -is(ch) konnte früher für ein und dieselbe Person gleichzeitig verwendet werden; das polnischprachige Bujaków bevorzugte aber die -us-Form.

17. Jh.: Hankeß, Hankißowa 1658, Hankis 1660, 1662, 1663, Hankusik (in Bujaków 1660, Gregor Hankus Vogt in Bujakow 1660), Hankus Lukas Ober-Seibersdorf 1661, Hankiß Bartholomäus und Susanna 1662, 1664 vgl. Hąnxa Bartholomäus, Ankisz Bartholomäus 1667, Hunkiss 1662, Ankus 1668.

Vor der Auswanderung: Honkis 1768

Unmittelbar nach der Auswanderung nach Anhalt, 1770: Hoinkes

Die polnischen Pfarrer schrieben Endungen mit -is oder -isch nebeneinander. Das verschwindende Anlauts-h ist eine polnische Erscheinung, wo es in Lehrwörtern Formen mit Anlauts-h und ohne diesen Laut (und Buchstaben) gab (vgl. bis heute gängige dialektale Formen hareszt, harmata, Hanka). Die Unsicherheit des polnischen Schreibers ist auch in der häufigen Schreibung des Vornamens Helias sichtbar.

Nach 1870: Hoinkis

Nach der Auswanderung nach Preußen wurde Hoinkes geschrieben. Kurz nach der Ankunft der Siedler schrieb ein Beamter der Herrschaft Pless Hońkis, wahrscheinlich in der Überzeugung, die aus Polen stammenden Siedler seien als Polen zu behandeln, ungeachtet ihres ethnischen Hintergrundes.

Herma

Der Name ist die dialektale Form des altdeutschen Vornamens Hermann.

17. Jh.: Herma 1660, 1682

Vor der Auswanderung: Herma 1764

Unmittelbar nach der Auswanderung nach Anhalt, 1770: Herma

Nach 1870: Herma

Klotz

Ein Übername in Form des bis heute verwendeten Substantivs Klotz. Da das Wort ins Polnische entlehnt wurde, sehr oft in der Diminutivform klocek, wurde es auch auf die deutschsprachigen Seibersdorfer Träger dieses Namens übertragen.

17. Jh.: Klocz Andreas und Katharina 1661 - vgl. Kłoczek Andreas und Katharina 1664

Vor der Auswanderung: Klocek 1764, Klocki 1756

Unmittelbar nach der Auswanderung nach Anhalt, 1770: Klotzek, Klotzky

Nach 1870: Klotzky

Durch die Preußen nicht ,richtiggestellt“, wahrscheinlich schien es ihnen ein rein polnischer Name zu sein, da ja die Siedler aus Polen kamen, was in allen Dokumenten vermerkt wurde.

Komendera 
Ein Übername zu kirchenlateinisch commendarius „Verwalter einer Pfarrei““ ${ }^{12}$ 17. Jh.:

Vor der Auswanderung: Komedera 1767

Unmittelbar nach der Auswanderung nach Anhalt, 1770: Commandeur

Nach 1870: Komendera

Kurz nach der Einwanderung in Preußen wurde der Name mit dem französischem Fremdwort assoziiert. Dann aber wurde wahrscheinlich die Abwegigkeit dieser Annahme bemerkt und durch den bekannten kirchlichen Terminus ersetzt.

Kunis

Eine Koseform zu Konrad. Der Name war schon 1584 in Seibersdorf belegt. ${ }^{13}$ 17. Jh.: Kunyss 1664, Kunis 1695

An der Auswanderung nach Anhalt nahmen keine Träger dieses Namens teil.

\section{Lenner}

Der Name wurde von den polnischen Schreibern stark entstellt. Eine Koseform zu Leonhard. Linnert und Lenner waren häufige Vornamen im 16. und 17. Jh. in Bielitz. ${ }^{14}$ Das unbegründete -i- war häufiges Merkmal der Polonisierung deutscher Namen, etwa Fugger zu Fukier.

17. Jh.: Lanier 1658, Linner 1658, Lenier 1660, Lenner 1660, Leyner Matthias, Susanna 1662 vgl. Susanna Ligierowa 1663, Leyner Georg und Eva 1664 - vgl. Lingier Georg und Eva 1667,

Lingier Gertrude 1662 - vgl. Gertrudis Linierowa 1663, Leynier 1665.

An der Auswanderung nach Anhalt nahmen keine Träger dieses Namens teil.

Mach

Wohl kein deutscher Name, sondern ein polnischer - eine Koseform von Matthias. ${ }^{15}$

Vor der Auswanderung: Mach 1759

Unmittelbar nach der Auswanderung nach Anhalt, 1770: Mach

Nach 1870: nicht belegt.

Mansler

Wohl eine Nebenform zu Menzler, was eine Kurzform von Hermenzel ist, das ist wiederum Hermann. ${ }^{16}$

${ }^{12} \mathrm{http}: / /$ genepedia.pl/index.php?title $=\mathrm{S} \% \mathrm{C} 5 \% 82$ ownik_funkcji_ko\%C5\%9Bcielnych_\%C5\%82ac.-pol. (25.09.2017).

${ }^{13}$ Kuhn (1981: 92).

14 Kuhn (1981: 92).

15 Bahlow (1953: 61).

16 Bahlow (1953: 45), Kuhn (1981: 92). 
17. Jh.: Mandzler 1660, Manczler Michael 1661 - vgl. Mandzler Michael 1664, Mandzer Michael 1667

Vor der Auswanderung, Mitte des 18. Jh: Mandzla 1770

Unmittelbar nach der Auswanderung nach Anhalt: Mansla oder Mansel

Nach 1870: Mansel

Obwohl der Anhalter Vogt Johann Mandzla seinen Namen auf eine so polonisierte Weise schrieb (Beleg vom 22. November $1781^{17}$ ), gewann eine „normal deutsch klingende" Form - Mansel.

Maier

Einer der häufigsten deutschen Namen schlechthin. Maier - 'Gutsverwalter' 17. Jh.: Maierowa 1668, Maier 1672

An der Auswanderung nach Anhalt nahmen keine Träger dieses Namens teil.

Manta

Wohl zu Mann in der Bedeutung 'Lehensmann'. ${ }^{18}$

17. Jh.: Manner Thomas 1660, Mander 1684, Manta Simon 1681

An der Auswanderung nach Anhalt nahmen keine Träger dieses Namens teil.

Nitsch

Einer der häufigsten Familiennamen der Deutschen Sprachinsel Bielitz, abgeleitet vom Vornamen Nikolaus. Seibersdorf war ein Stammort der Nitsch, wo der Name schon 1584 belegt ist. ${ }^{19}$

17. Jh.: Nycz 1658, Nicz 1659, Nytz Elias 1660 - vgl. Elias Nycz 1663, Necowa 1662

An der Auswanderung nach Anhalt nahmen keine Träger dieses Namens teil. Nicz).

Die polnischen Schreiber neigten zur Polonisierung der Schreibung (Nycz,

Obricht

Eine schlesische Form des Vornamens Albrecht. ${ }^{20}$

17. Jh.: Olbricht (Olbrychcianka) 1658, Olbert 1662

An der Auswanderung nach Anhalt nahmen keine Träger dieses Namens teil.

Schwarz

Ein Übername.

17. Jh.: Sworcz, Swartz 1659, Szwartz 1660, Swocowa 1661.

An der Auswanderung nach Anhalt nahmen keine Träger dieses Namens teil.

\footnotetext{
17 Staatsarchiv Kattowitz, Abteilung Pless, Bestand 53, Signatur XIII 607.

18 Bahlow (1953: 132).

19 Kuhn (1981: 92).

${ }^{20}$ Bahlow (1953: 51).
} 
Den polnischen Schreibern bereitete die Schreibung dieses Namens Probleme, zumal sie ihn wahrscheinlich nicht in der heutigen hochsprachlichen Form hörten. Im benachbarten Wilmesau heißt dieses Wort śwuö ${ }^{21}$ oder świöc. ${ }^{22}$ Der Dialekt der Seibersdorfer war dem Dialekt der Wilmesauer fast gleich. ${ }^{23}$ Die vom a abweichende Aussprache des einzigen Vokals und das vokalisierte r verursachten die schwankenden Schreibungen.

\section{Stefke}

Im 17. Jh. hatte dieser Familienname noch die dialektale Form des Namens Stefan mit dem für den Bielitzer Dialekt und das Schlesische im Allgemeinen typischen Verschiebung der Auslautendung -en zu a (vgl. Murga 'Morgen', surga 'sorgen', bezohla 'bezahlen'). ${ }^{24}$ Dann wurde ein k-hältiges Suffix angehängt, möglicherweise durch Analogie zu vielen anderen Familiennamen dieser Art in der Sprachinsel, etwa Perschke, Berke, Thomke, Snatschke, Urbanke.

17. Jh.: Sztafa Lucas und Katharina 1668, auch Stafa 1670

Vor der Auswanderung: Stefko 1768

Unmittelbar nach der Auswanderung nach Anhalt, 1770: Steffke, Stewki

Nach 1870: Stefke

Die polnischen Schreiber schrieben den Namen mit auslautenden -o, möglicherweise durch Analogie zu häufigen polnischen Koseformen der männlichen Vornamen mit der Endung -ko, etwa Janko, Jaśko, Maćko, Staśko usw. In Preußen kehrte man zu der typischen (nord)deutschen Endung der Familiennamen mit -ke zurück.

\section{Schön}

Ein Übername.

17. Jh.: Szyn 1661, 1668, Szyen Simon 1697, Szęn 1698.

Vor der Auswanderung: Schein 1766

Unmittelbar nach der Auswanderung nach Anhalt: Schoen

Nach 1870: Schön

Die Schreibung dieses Namens hat viele Versionen, aber auch die lokale dialektale Aussprache weicht von der heutigen standardsprachlichen ab: schein, ${ }^{25}$ śyjn, ${ }^{26}$ siejn. ${ }^{27}$ Die polnischen Schreibungen versuchen, dieser diphthongischen

${ }^{21}$ Latosiński (1909: 285).

22 Gara: https://upload.wikimedia.org/wikipedia/commons/d/d0/J\%C3\%B3zef_Gara_-_S\%C5\% 82ownik_j\%C4\%99zyka_wilamowskiego.pdf (26.09.2017).

23 Wackwitz (1932: 14).

${ }^{24}$ Bukowski (1860: 123).

25 Bukowski (1860: 178).

${ }^{26}$ Latosiński (1909: 294).

27 Gara: https://upload.wikimedia.org/wikipedia/commons/d/d0/J\%C3\%B3zef_Gara_-_S\%C5\% 82ownik_j\%C4\%99zyka_wilamowskiego.pdf(26.09.2017). 
Aussprache Rechnung zu tragen. In Preußen wurde die Schreibung dieses Namens hochsprachlich.

\section{Sonntag}

Ein Übername.

17. Jh.: Zontak 1686

Vor der Auswanderung: Zontak 1766

Unmittelbar nach der Auswanderung nach Anhalt: Sonntag

Nach 1870: Sonntag

Die polnischen Schreiber schrieben den Namen phonetisch mit polnischen Schriftzeichen, die Preußen nach korrekter deutscher Rechtschreibung.

\section{Wagner}

Eine Berufsbezeichnung.

17. Jh.: Wanier 1660, Vanier/Wanier Johann und Anna 1689, 1692

Vor der Auswanderung: Wanier 1766

Unmittelbar nach der Auswanderung nach Anhalt, 1770: Waneer korrigiert auf Wagner

Nach 1870: Wagner

Nach Bahlow (1953, S. 119) waren die zusammengezogenen dialektalen Formen, etwa Weiner, Wähner, Wehner(t) im Schlesischen recht verbreitet. So muss der Name auch in Seibersdorf ausgesprochen worden sein, denn die vor der Auswanderung von den polnischen Geistlichen aufgezeichneten Formen beinhalten nie das g-Zeichen. Dazu kommt die schon erwähnte polnische Sitte, die deutschen Endungen -er mit einem -i- auszustatten (-ier). Nach der Auswanderung nach Preußen wurden die Wanniers zu Wagners korrigiert, was im ersten Verzeichnis der Kolonisten sichtbar wird. ${ }^{28}$

Weiß

Ein Übername.

17. Jh.: Weyszowa Susanna 1662, Weyßtomasowa Susanna 1663, Biały Thomasz 1667, Weysz Thomas und Susanna 1674

Vor der Auswanderung: Weis 1754

An der Auswanderung nach Anhalt nahmen keine Träger dieses Namens teil. Interessant ist nicht die Schreibung des Namens selbst, sondern die vereinzelten Übersetzungen dieses Namens ins Polnische als „Biały”.

Wiesner

Ein Wohnstättenname.

17. Jh.: Wyzner Georg 1680, 1681

An der Auswanderung nach Anhalt nahmen keine Träger dieses Namens teil.

${ }^{28}$ Staatsarchiv Kattowitz, Abteilung Pless, Bestand 53, Signatur XIII 607. 
Die polnischen Schreiber schrieben den Namen mit ,Z“, was der polnischen regressiven Assimilation von [s] zu [z] vor dem Stimmhaften [n] entspricht. Der bis heute verbreitete Name wird ['vizner] ausgesprochen, und nicht wie im Deutschen ['vi:snb].

\section{Zänger}

Der Name war sonst in der Sprachinsel nicht bekannt. Die Belege aus dem 17. Jh. beinhalten nie das Zeichen ,g“" im Namen, die Grundlage dürfte die mhd. Berufsbezeichnung zeiner sein (Schmied) ${ }^{29}$. Das mhd. Adjektiv zanger oder zenger bedeutete munter, lebhaft, rührig ${ }^{30}$.

17. Jh.: Zeiner, Ceiner 1659), Cenier 1661, Czenner Andreas, Susanna 1661, 1664 - vgl. Susanna Czeynerowa 1664, 1666, Andreas Cenier 1672, Susanne Cenierowa, Cenier Andreas und Susanna 1672. Cener Simon, Anna 1661 - vgl. Cenier Simon 1668, Cener Jerzy 1661, Cenier Simon und Susanna 1669, 1681, Cener Andreas 1697, Celner Bartholomäus 1697, Celnerka Susanne 1697, Czynier Johann und Susanna 1696 - vgl. Cenner Johann und Susanna 1697, Czeyner Simon und Eva 1663,

Zenger Elias Müller, Andreas 1660, Susanna 1661

Vor der Auswanderung: Cenier 1769, Zyngar 1768

Nach der Auswanderung nach Anhalt: Zenger

Nach 1870: Zänger

Abgesehen von dem ,polnischen“-i- in den Schreibungen des 17. Jh., bereitet dieser Name dem Forscher auch andere Schwierigkeiten. Es sind nämlich mehrere Grundlagen möglich, auch die Herkunft von Zaun ist nicht auszuschließen. Die preußische Schreibung mit „ä“ kann als eine Volksetymologie bezeichnet werden, da hier anscheinend als Grundlage „Zange“ angenommen wurde.

\section{Allgemeine Tendenzen der Entwicklung der Familiennamen}

\subsection{Vor der Auswanderung der Seibersdorfer}

Die Namen der Seibersdorfer sind uns vor allem aus den Kirchenmatrikeln der dortigen katholischen Pfarrei bekannt, die sich auch für die einheimischen deutschsprachigen Protestanten zuständig fühlte. Wie die Aufzeichnung von Taufen, Eheschließungen und Begräbnissen in Wirklichkeit funktionierte, wissen wir heute nicht mehr. Die Beziehungen zwischen der protestantischen Bevölkerung und der katholischen Kirche und Obrigkeit gelten in der Literatur als schlecht ${ }^{31}$, was die deutschsprachigen Seibersdorfer schließlich zur Auswanderung bewog.

\footnotetext{
${ }^{29}$ Lexer (1962: 331).

${ }^{30}$ Lexer (1962: 330).

${ }^{31}$ Kuhn (1982: 250).
} 
$\mathrm{Ob}$ es zu einem persönlichen Kontakt zwischen dem polnischen Pfarrer und den deutschen Einwohnern bei solchen Anlässen kam, ist nicht bekannt. Für manche Zeiträume kann als sicher gelten, dass die Protestanten geheime Gottesdienste im Gebirge abhielten.

Als sicher kann auch gelten, dass die polnischen Schreiber (wahrscheinlich die Geistlichen selbst) die Namen der deutschen Bevölkerung phonetisch nach den Regeln der polnischen Rechtschreibung schrieben, ohne sich die Mühe zu geben, sie richtig auf Deutsch zu schreiben. Die meisten Einwohner von Seibersdorf waren des Lesens und Schreibens unkundig, so dass es keine Tradition der Namensschreibung gab. Dies erklärt die Schwankungen in der Namensschreibung. Antworten auf ihre Fragen nach dem Namen kamen sicher im Dialekt, da die Seibersdorfer ja nur des Dialekts mächtig waren. Die Unkenntnis der Schriftsprache konservierte diese Formen.

\subsection{Nach der Niederlassung in Anhalt}

Nach der Auswanderung nach Preußen wurden die „Missstände“ in der Namensführung von Amts wegen beseitigt. Die preußischen Beamten versuchten, die Namensformen an die ihnen bekannten schriftsprachlichen Formen anzupassen. Eine Siedlerliste mit nachträglichen Streichungen und Korrekturen der Namensschreibungen ist ein Beweis dafür. In manchen Fällen war dieses umstrittene Vorgehen erfolgreich und methodologisch richtig. Nimmt man an (egal, ob das begründet ist), dass die Familiennamen sich an schriftsprachlichen Formen orientieren sollen, so ist eine Berichtigung wie Bayger zu Berger gerechtfertigt. Die Beamten waren aber keine Philologen und ließen sich in manchen Fällen zu Volksetymologien verleiten. Sie entzifferten beispielsweise den Namen Englert als Engel, während es sich um den alten deutschen Rufnamen Engelhard handelt. Auf diese Weise entstanden Anhalter Parallelformen zu Namen aus der Deutschen Sprachinsel Bielitz.

\section{Schlusswort}

Die deutsche Sprachinsel Anhalt-Gatsch existiert seit der Vertreibung der deutschen Bevölkerung nach dem Zweiten Weltkrieg nicht mehr. Nach über siebzig Jahren sind so gut wie keine Sprecher des dortigen Dialekts auffindbar. Sollten noch welche leben, die als Kinder das Dorf verließen, so sind sie in ihrer neuen Umgebung in Deutschland sozialisiert und können nicht als Probanden gelten. Deswegen können nur schriftliche Quellen Informationen zu dortigen Dialektverhältnissen liefern. Dieser Beitrag soll auch als Nachweis dienen, dass das Studium alter Dokumente zusammen mit zufällig aufgezeichneten Erinnerungen (in 
unserem Fall des Pastors Wackwitz) Aufschluss über Dialektverhätnisse in längst eingegangenen Sprachinseln geben kann. Die Familiennamen der Einwohner von Sprachinseln tragen, wie man hier sehen kann, der dialektalen Aussprache Rechnung. Spätere Quellen können unter Umständen amtliche „Verschlimmbesserungen" aufweisen.

\section{Literatur}

\section{Quellen}

Archiwum Państwowe w Katowicach, Oddział w Pszczynie (Staatsarchiv Kattowitz, Filiale Pless), Bestand Archwum Książąt Pszczyńskich [Archiv der Fürsten von Pless], Signatur XIII 606, 607; Bestand Landratura [Landratsamt], Signatur 122.

Kirchenmatriken der katholischen Pfarrei Kozy: Liber Matrimonium Contrabtorum [...] 1658, Metrica Baptisatorum 1658-1692, Matrica Natorum et Mortuorum ab Anno 1751-1772, Tomus IV.

Kirchenmatriken der reformierten Pfarrei Anhalt: Begräbniß-Buch No. 2, 1795-1837 auf: http://siliusradicum.pl/ksiegi-metrykalne/asc-parafii-ewangelickiej-w-holdunowie-anhalt/ (28.09.2017).

Webseite der Freiwilligen Feuerwehr Kozy: http://www.osp.kozy.pl (28.09.2017).

Website des Gimnazjum im Jana Pawła II in Kozy: http://gimnazjum.nexcom.pl/index.php/olimpiady-i-konkursy (28.9.2017).

\section{Bearbeitungen, Nachschlagewerke}

Bahlow, Hans (1953): Schlesisches Namenbuch. Kitzingen/Main.

Bukowski, Jacob (1860): Gedichte in der Mundart der deutschen schlesisch-galizischen Gränzbewohner, resp. von Bielitz-Biala. Bielitz.

Gara, Józef: „Słownik języka wilamowskiego” przetłumaczono na język: polski, niemiecki, angielski. Auf: https://upload.wikimedia.org/wikipedia/commons/d/d0/J\%C3\%B3zef_Gara__S\%C5\% 82ownik_j\%C4\%99zyka_wilamowskiego.pdf (26.09.2017).

Kuhn, Walter (1981): Geschichte der deutschen Sprachinsel Bielitz (Schlesien). Würzburg.

Malina, Adam (1994): Ewangelickie tradycje Hołdunowa. Katowice.

Latosiński, Józef (1909): Monografia miasteczka Wilamowic. Kraków.

Lexer, Matthias (1962): Mittelhochdeutsches Taschenwörterbuch. Leipzig.

Wackwitz, Andreas (1932): Die deutsche Sprachinsel Anhalt-Gatsch in Oberschlesien in ihrer geschichtlichen Entwicklung. Plauen im Vogtland. 